\title{
A Case Report of Arcuate Uterus
}

\author{
K Fatema
}

\begin{abstract}
A uterine malformation is the result of an abnormal development of the Mullerian duct(s) during embryogenesis. The arcuate uterus is a type of congenital uterine malformation where the uterine fundus displays a small midline indentation towards the uterine cavity. A 25 years old woman with history of two abortions was diagnosed as a case of arcuate uterus during her first caesarean section. With proper antenatal care and counseling she has been able to give birth to 2nd baby successfully. No correctable surgery was essential for her. The literature regarding the diagnosis, management, and reproductive outcomes for arcuate uterus is limited and conflicting. A woman with an arcuate uterus can carry a baby to full term pregnancy. However, this condition is associated with a higher risk for miscarriage and premature births. Arcuate uterus is usually managed similarly to septate uterus, and only selected patients who fulfill poor reproductive performance criteria are recommended for surgical correction.
\end{abstract}

Key words: Arcuate uterus, Malformation, Mullerian duct.

\section{Introduction :}

The uterus is formed during embryogenesis by the fusion of the two mullerian ducts. During this fusion a resorption process (begins caudally and advances cranially) eliminates the partition between the two ducts to create a single cavity. The arcuate uterus is a form of a uterine anomaly or variation where the uterine fundus displays a concave contour towards the uterine cavity that has an apical angle of more than 90 degrees results from near-complete resorption of the uterovaginal septum, but the causes for incomplete resorption are not known. Normally the fundus of the uterus is straight or convex on anterior-posterior imaging but the arcuate uterus contains a residual cranial septum that is smaller than an incomplete septum.

An arcuate uterus compared with other mullerian malformations, is clinically benign despite an infrequent association with adverse obstetric outcomes, and may not affect reproductive outcomes ${ }^{1}$. The therapeutic approach in mullerian duct anomalies is specific to the type of anomaly and includes non-treatment, medical therapy and surgical treatment. It is important to carry out comprehensive examinations and to be certain that the fertility and obstetric problems stem purely from uterine causes before taking surgical steps to correct those problems.

\footnotetext{
DrKaneez Fatema, MS (Obst \& Gynae), Assistant Professor, Dept of Obstetrics and Gynaecology, FMC, Faridpur.

Address of correspondence

Dr Kaneez Fatema, MS (Obst \& Gynae), Assistant Professor, Dept of Obstetrics and Gynaecology, FMC, Faridpur. Phone: +88-0171-167362;

E-mail: moyourakkhi2003@yahoo.com
}

\section{Case report:}

Farzana, a 25-year-old woman, a housewife of a middle socio-economic class was in regular antenatal check-up as a case of high risk pregnancy. She had history of two spontaneous abortions followed by sub-fertility for 3 years and became pregnant after ovulation induction. The baby was presented by breech but at her 33 weeks of pregnancy she had sudden premature rupture of membrane and she was admitted to a private hospital where she had a lower uterine segment caesarean section and an arcuate uterus was discovered intraoperatively by visual examination and palpation. The pelvic examination revealed a single cervix and no vaginal septum. A premature female baby of $2 \mathrm{~kg}$ weight was managed by an expert neonatologist just after delivery. There was no further workup in the postpartum period.

During her second pregnancy after 2 years, again she was in regular check-up and the baby had transverse presentation. The patient was given strict precautions to report to the hospital in case of any problem or abdominal pain. She was planned for elective caesarean section on 1st November, 2010 which was two weeks prior to the expected date of delivery (EDD) but she failed to come on the selected date. On 3rd November 2010 at her 38 weeks of pregnancy, she rushed Faridpur with labour pain and an emergency caesarean section was performed and impending rupture was detected during operation. A healthy male baby of $3 \mathrm{~kg}$ was delivered. 


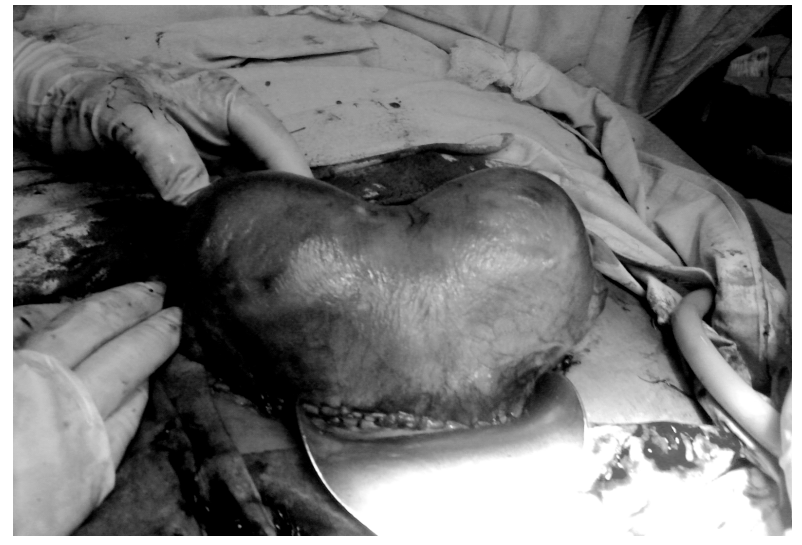

Fig.1: Per-operative view of arcuate uterus following caesarean section

\section{Discussion :}

Normal development of the female reproductive tract involves a series of highly orchestrated, complex interactions that direct differentiation of the mullerian ducts and urogenital sinus to form the internal female reproductive tract. When an interruption or dysregulation occurs in any of the dynamic processes of differentiation, migration, fusion and canalization, a wide spectrum of mullerian duct anomalies can result ${ }^{1,2}$.

The prevalence of uterine malformation is estimated to be $6.7 \%$ in the general population, slightly higher $(7.3 \%)$ in the infertility population, and significantly higher in a population of women with a history of recurrent miscarriages $(16 \%)^{3}$. The American Fertility Society classifies uterine malformations into seven separate categories (Table I) which is most widely accepted. Their frequency distribution is septate in 34\%, bicornuate in $29 \%$, didelphic in $11 \%$, arcuate in $7 \%$, unicornuate in 5\% and hypoplastic/aplastic and other forms in $4 \%$ cases $^{4}$.

Table I: American Fertility Society Classification of Uterine Malformations

\begin{tabular}{cl}
\hline Class & \multicolumn{1}{c}{ Uterine malformations } \\
\hline I & Hypoplasia/ uterine agenesis \\
II & Unicornuate uterus \\
III & Uterus didelphys \\
IV & Bicornuate uterus \\
V & Septate uterus \\
VI & Arcuate uterus \\
VII & T - shaped uterus resulting from the use of \\
& Diethylstilbestrol \\
\hline
\end{tabular}

Women with congenital anomalies of the genital tract may be asymptomatic, but generally they can suffer a wide range of symptoms, which can manifest themselves at any of the various stages of life, from
Women with congenital anomalies of the genital tract may be asymptomatic, but generally they can suffer a wide range of symptoms, which can manifest themselves at any of the various stages of life, from childhood to senescence; before pregnancy, during pregnancy and after pregnancy. Approximately 25\% of women with mullerian anomalies have fertility and obstetric problems as compared with $10 \%$ of the normal healthy population ${ }^{5}$. There is generally no problem in conceiving but rather in maintaining a normal pregnancy to its completion. Still, the incidence of unexplained infertility among these cases could be as high as $35 \%$. Spontaneous abortion in the first and second trimester of pregnancy, abnormal fetal presentation, premature birth, dystocia, pathologic conclusions of pregnancy, stillbirth, and ectopic pregnancy are among the many problems that appeared during pregnancy and labour.

A pelvic examination will not reveal the condition. Investigations are usually prompted on the basis of reproductive problems. Helpful techniques to investigate the uterine structure are transvaginal ultrasonography and sonohysterography, hysterosalpingography (HSG), magnetic resonance imaging (MRI), and hysteroscopy. HSG reveals a single uterine cavity with a saddle-shaped fundal indentation. MRI findings show convex or flat external uterine contour. The indentation is broad and smooth. More recently 3-D ultrasonography has been advocated as an excellent non-invasive method to delineate the condition ${ }^{6}$.

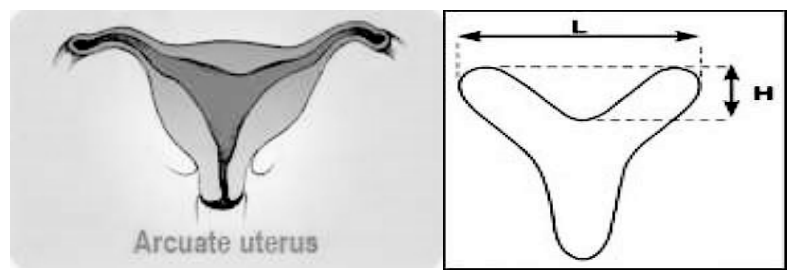

Fig. 2: A diagrammatic representation of Arcuate Uterus

The arcuate uterus is normal in shape with a small midline indentation of the uterine cavity. The indentation results from the failure of the median septum to completely dissolve. This uterine anomaly is essentially normal, but is still given a distinct class. Many patients with an arcuate uterus will not experience any reproductive problems and do not require any surgery. Other views are that the condition is associated with a higher risk for miscarriage, premature birth, and malpresentation. Woelfer found that the miscarriage risk is more pronounced in the second trimester ${ }^{6}$.

Uterine malformations although less common but not rare in Bangladesh. Most studies of uterine malformations are based on populations of women who have experienced a pregnancy loss and thus do not 
address the issue of the prevalence in the general population. A screening study by Woelfer et al. of women without a history of reproductive problems found that about $5 \%$ of women had arcuate uterus which was the most common uterine anomaly, followed by uterine septum $(3 \%)$ and bicornuate uterus $(0.5 \%)^{6}$. In contrast, in about $15 \%$ of patients with recurrent pregnancy loss anatomical problems are thought to be causative with the septate uterus as the most common finding ${ }^{7}$.

The presented subject is a young female of 25 years had history of two spontaneous abortions then a variable duration of infertility followed by two pregnancies. During her first pregnancy she had premature labor and in both pregnancies she had malpresentation, initially breech later transverse. Reproductive outcome data in this condition are conflicting and both positive and negative outcomes have been reported $^{8}$. In a retrospective case series of 176 patients, Acien P. reported a $45 \%$ early abortion rate in women with arcuate uterus ${ }^{9}$. In contrast, Raga et al, in their series noted only a $13 \%$ early miscarriage rate in women with this anomaly. Treatment is usually expectant ${ }^{10}$. Combined data from various studies that included reproductive outcomes for the arcuate uterus were comprehensively reviewed in one report. No uniformity was observed with respect to data included for live birth rates, delivery rates and ectopic pregnancy rates, rendering interpretation of following reproductive outcome results of 283 difficult pregnancies:10 (5.1\%) of 195 preterm deliveries, $129(66.2 \%)$ of 195 live births, 7 (3.6\%) of 195 ectopics, and 57 (20.1\%) of 283 spontaneous abortions ${ }^{11}$.

A study based on hysterosalpingoraphy detected arcuate lesions documented increased fetal loss and obstetrical complications as a risk for affected women ${ }^{12}$. In contrast, a study utilizing 3-D ultrasonography to document the prevalence of the arcuate uterus in a gynaecological population found no evidence of increased risk of reproductive loss; in this study $3.1 \%$ of women had an arcuate uterus making it the most common uterine anomaly ${ }^{5}$. Many patients with an arcuate uterus will not experience any reproductive problems and do not require any surgery. In patients with recurrent pregnancy loss thought to be caused by an arcuate uterus, hysteroscopic resection can be performed as the intervention, is relatively minor and safe in experienced hands. A follow-up imaging study should demonstrate the removal of the lesion.

\section{References}

1. Heinonen PK. Complete septate uterus with longitudinal vaginal septum. Fertil Steril. 2006; 85(3):700-5.

2. Raziel A, Arieli S, Bukovsky I, Caspi E, Golan A. Investigation of the uterine cavity in recurrent aborters. Fertil Steril. 1994; 62(5):1080-1082.

3. Saravelos SH, Cocksedge KA, Li TC. Prevalence and diagnosis of congenital uterine anomalies in women with reproductive failure: a critical appraisal. Human Reproduction 2008; 14(5):415-29.
4. The American Fertility society classifications of adnexal adhesions, distal tubal occlusion, tubal occlusion secondary to tubal ligation, tubal pregnancies, mullerian anomalies and intrauterine adhesions. Fertil Steril. 1988; 49(6):944-55.

5. Jurkovic D, Gruboeck K, Tailor A, Nicolaides KH. Ultrasound screening for congenital uterine anomalies. Br J Obstet Gynaecol. 1997; 104(11):1320-1.

6. Woelfer B, Salim R, Banerjee S, Elson J, Regan L, Jurkovic D. Reproductive outcomes in women with congenital uterine anomalies detected by three-dimensional ultrasound screening. Obstet Gynecol. 2001; 98(6):1099-103.

7. Propst AM, Hill JA. Anatomic factors associated with recurrent pregnancy loss. Semin Reprod Med. 2000; 18(4):341-50.

8. Troiano RN. Magnetic resonance imaging of mullerian duct anomalies of the uterus. Top Magn Reson Imaging 2003; 14(4):269-279.

9. Acien P. Reproductive performance of women with uterine malformations. Hum Reprod. 1993; 8:122-126.

10.Raga F, Bauset C, Remohi J, Bonilla-Musoles F, Simon C, Pellicer A. Reproductive impact of congenital Mullerian anomalies. Hum Reprod. 1997; 12(10):2277-2281

11.Lin PC. Reproductive outcomes in women with uterine anomalies. J Womens Health 2004; 13(1):33-9.

12.Sørensen SS, Trauelsen AG. Obstetric implications of minor mullerian anomalies in oligomenorrhoeic women. Am J Obstet Gynecol. 1987; 156 (5):1112-8. 